\title{
Acquired hypohidrosis following a drug reaction
}

A 44-year-old Chinese man presented with a 3-week history of heat intolerance and a reduced ability to sweat even upon strenuous physical exertion. His medical history was significant for HIV infection on treatment with efavirenz and lamivudine/zidovudine. He also had drug reaction with eosinophilia and systemic symptoms (DRESS) to Bactrim 14 months prior, during which he developed a generalised exanthem. Physical examination revealed skin-coloured papules on his entire trunk with no visible beads of sweat despite having walked a distance in the afternoon heat to the consult room (Fig. 1). A skin biopsy was performed on one of the papules (Fig. 2). What is your diagnosis?
A. Cholinergic urticaria
B. Folliculitis
C. Miliaria profunda
D. Molluscum contagiosum
E. Pruritic papular eruption of HIV

The physical examination findings of skin-coloured papules on the trunk with no visible beads of sweat is suggestive of miliaria profunda (MP). The skin biopsy showed dilated acrosyringia with chronic lymphohistiocytic infiltrates surrounding superficial portions of the eccrine sweat ducts (Fig. 2). A starchiodine sweat test demonstrated absence of sweat on our patient's trunk and limbs, but normal sweat response on his neck and face. High-definition optical coherence tomography (HD-OCT) showed features consistent with MP (Fig. 3). He was diagnosed with MP and acquired hypohidrosis, as a possible late complication of DRESS. The patient declined specific treatment, and was advised to avoid heat and strenuous activities. His symptoms eventually resolved over a year, and he was able to resume his work as an odd job labourer.

Miliaria refers to the cutaneous obstruction and disruption of eccrine sweat glands. It can be sub-classified into 3 main types (crystallina, rubra and profunda) based on the depth of disruption within the sweat duct, and these can be differentiated by clinical appearance and histology. MP manifests with skin-coloured papules that appear when the patient is hot and tries to sweat, and resolves gradually as the patient cools down. The leakage of sweat into the papillary dermis can cause substantial periductal lymphocytic infiltrate and spongiosis of the eccrine ducts, which are histological

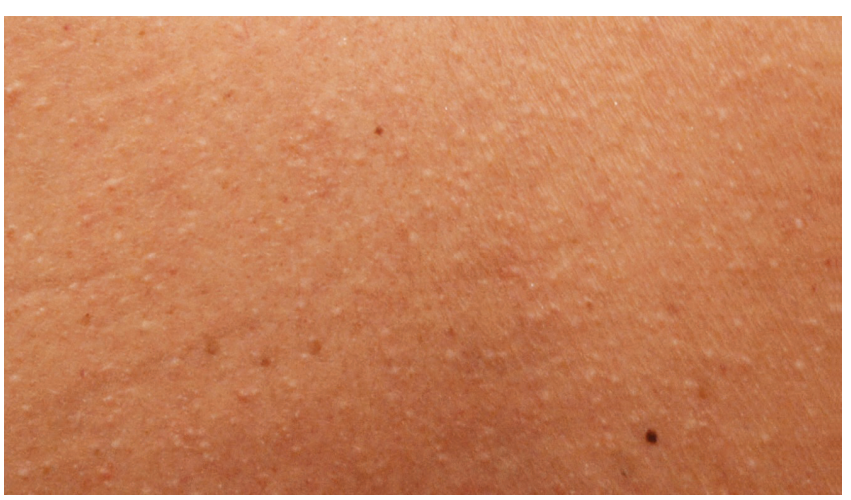

Fig. 1. Multiple skin-coloured dermal papules over the back.

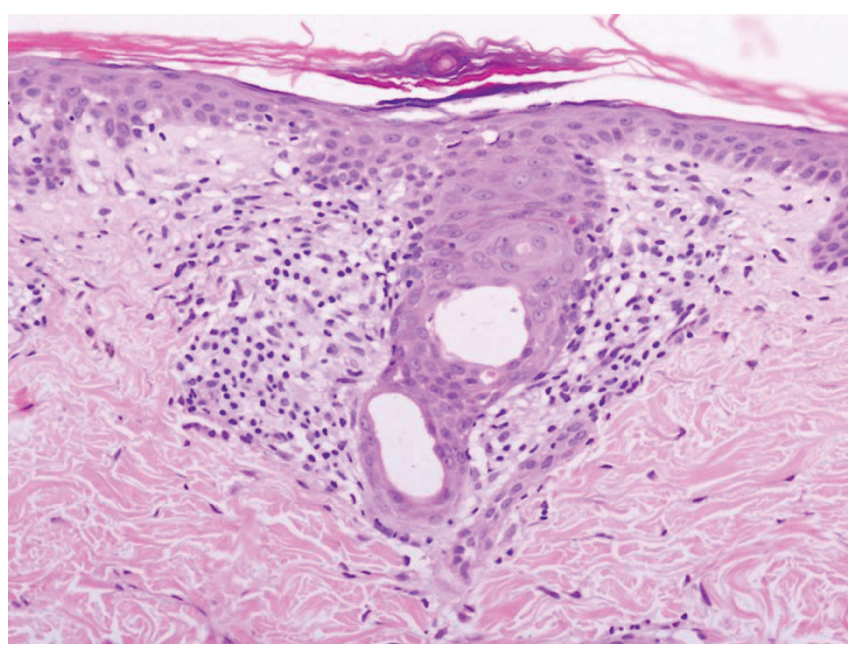

Fig. 2. A chronic lymphohistiocytic infiltrate surrounds the superficial portions of a dilated eccrine duct. (H\&E, X200)

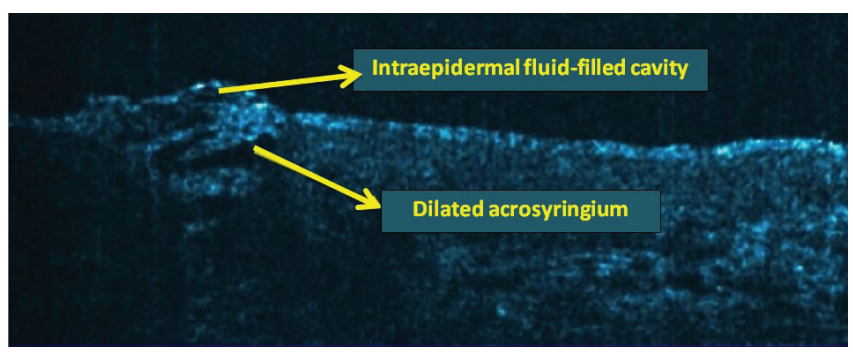

Fig. 3. Optical coherence tomography after starch iodine sweat test showed an intraepidermal fluid-filled cavity and dilated acrosyringium in an area of anhidrosis on the trunk.

features of the disease. In this case, the physical examination findings and subsequent histological analysis are consistent with MP. 
The different diagnoses listed do not cause reduced sweating but need to be considered. Cholinergic urticaria is a common condition that occurs when there is an increase in body temperature, and presents with itchy, small, erythematous, urticarial papules predominantly on the trunk and arms. It is transient and resolves as the body temperature cools to normal. Folliculitis can be recognised by the presence of papules of an erythematous, follicular-centred nature, which was not seen in this patient. Molluscum contagiosum is a poxvirus infection, which is more common in immunocompromised patients, and presents with umbilicated skin-coloured papules with a pearly appearance. Pruritic papular eruption of HIV is characterised by intense pruritus, and manifests with chronic papules that are often eroded from persistent scratching. The skin lesions in our patient were not pruritic.

DRESS is known to be associated with a wide range of complications. Acute life-threatening complications include liver, renal or cardiopulmonary dysfunction, or sepsis due to immunosuppression. Delayed-onset complications occur in about $10 \%$ of patients, and develop within 2 months to 3 years of the disease onset. Autoimmune sequelae include thyroid dysfunction, diabetes mellitus type 1, polyglandular autoimmune syndromes, systemic lupus erythematosus, autoimmune haemolytic anaemia and autoimmune enteropathy. Dermatological autoimmune sequelae such as alopecia areata and vitiligo have also been reported. ${ }^{1,2}$

Hypohidrosis refers to a diminished sweating response to an appropriate stimulus. Physiologically, humans sweat more over certain regions of the body, such as the face, axillae, palms and soles. The trunk and legs are usually affected earlier and more often in hypohidrosis, which was the case in our patient. Causes of hypohidrosis include various congenital diseases, connective tissue disorders and drugs. ${ }^{3,4}$ Hypohidrosis has also been reported to occur from the dysfunction of sweat pores secondary to chronic inflammatory dermatoses such as atopic dermatitis and psoriasis. ${ }^{3}$ Dilated spiraling acrosyringium with macerated keratin have been observed on HD-OCT, which corresponds to epidermal spongiosis and hyperkeratotic plugs at sweat orifices seen on histologic analysis. ${ }^{5}$ As these findings were also seen in our patient, we posit that his condition is caused by obstruction at the sweat orifices from hyperkeratotic plugs due to DRESS. In his case, DRESS had clinically appeared to have resolved 14 months prior.

This case demonstrates that MP and hypohidrosis can occur as a late complication in patients with DRESS. This has not been previously reported and is of importance as hypohidrosis is a potentially fatal condition that can have significant impact on a patient's work and lifestyle.

\section{REFERENCES}

1. Shiohara T, Mizukawa Y. Drug-induced hypersensitivity syndrome (DiHS)/drug reaction with eosinophilia and systemic symptoms (DRESS): An update in 2019. Allergol Int 2019;68:301-8.

2. Lian BS, Busmanis I, Lee HY. Relapsing course of sulfasalazineinduced drug reaction with eosinophilia and systemic symptoms (DRESS) complicated by alopecia universalis and vitiligo. Ann Acad Med Singap 2018;47:492-3.

3. Chia KY, Tey HL. Approach to hypohidrosis. J Eur Acad Dermatol Venereol 2013;27:799-804.

4. Chia BK, Chong WS, Tey HL. Generalised anhidrosis secondary to intracranial haemorrhage. Ann Acad Med Singap 2016;45:69-70.

5. Tey HL, Tay EY, Cao T. In vivo imaging of miliaria profunda using high-definition optical coherence tomography: diagnosis, pathogenesis, and treatment. JAMA Dermatol 2015;151:346-8.

Gareth Lim, ${ }^{1} M B B S, B S c$ (Hons),

Hui Yi Chia, ${ }^{2}$ MBBS, MRCP (UK), Dip Dermatopathology (ICDP-UEMS),

Hong Liang Tey, ${ }^{2,3,4}$ MBBS, FRCP (Edin), MRCPS (Glasg),

Yen Loo Lim, ${ }^{2}$ MBBS, MRCP (UK), FRCP (Edin),

Yee Kiat $\underline{\text { Heng, }}{ }^{2}$ MBBS, MRCP (UK), Med MMed (Int Med)

${ }^{1}$ Internal Medicine, Tan Tock Seng Hospital, Singapore

${ }^{2}$ National Skin Centre, Singapore

${ }^{3}$ Lee Kong Chian School of Medicine, Nanyang Technological University, Singapore

${ }^{4}$ Yong Loo Lin School of Medicine, National University of, Singapore

Correspondence: Dr Gareth Lim, Internal Medicine, Tan Tock Seng Hospital, 11 Jalan Tan Tock Seng, Singapore 308433.

Email: gareth.lim@mohh.com.sg 